Check for updates

Cite this: RSC Adv., 2018, 8, 13388

Received 10th March 2018

Accepted 29th March 2018

DOI: $10.1039 / c 8 r a 02138 d$

rsc.li/rsc-advances

\section{A simple two-photon turn-on fluorescent probe for the selective detection of cysteine based on a dual PeT/ICT mechanism $†$}

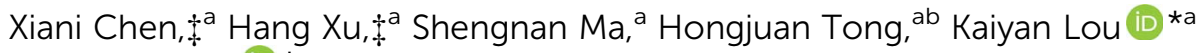 \\ and Wei Wang (iD *ac
}

Herein, a simple two-photon turn-on fluorescent probe, $N$-(6-acyl-2-naphthayl)-maleimide (1), based on a dual PeT/ICT quenching mechanism is reported for the highly sensitive and selective detection of cysteine (Cys) over other biothiols. The probe was applied in the two-photon imaging of Cys in cultured HeLa cells, excited by a near-infrared laser at $690 \mathrm{~nm}$.
Cysteine (Cys), homocysteine (Hcy), and glutathione (GSH) are structurally similar biothiols, but their biological functions are quite different from one another. ${ }^{1-6}$ Among these biothiols, Cys functions as one of the twenty-one amino acids for peptide and protein synthesis, and Cys deficiency is also associated with certain disease symptoms. ${ }^{7-10}$ Methods for the selective detection and differentiation of Cys among different biothiols, including high performance liquid chromatography (HPLC), ${ }^{11}$ capillary electrophoresis, ${ }^{12}$ electrochemical assay, ${ }^{13}$ UV-vis spectroscopy, ${ }^{14}$ and fluorescence-based methods, ${ }^{15-17}$ are important for its biological studies. Recently, fluorescent probes have attracted much attention as vital chemical biology tools due to their high sensitivity, convenient operation, and real-time imaging capabilities. ${ }^{18-20} \mathrm{~A}$ number of Cys-selective fluorescent probes have been reported, ${ }^{21}$ which utilize Cysselective recognition groups such as aldehydes ${ }^{11,22}$ acrylates ${ }^{23}$ thioesters ${ }^{24}$ and electron-deficient aromatic halides ${ }^{25-27}$ in their structures. However, many of them have relatively long response times and low sensitivity due to a slow cyclization process. In addition, fluorescent probes with high selectivity for Cys over Hcy are difficult to achieve because they differ by only one methylene group. ${ }^{28}$ Recently, we reported that $N$-( $N^{\prime}$-butyl1,8-naphthalimide-4-yl)-maleimide, containing a single maleimide group as the recognition group, is a fast, sensitive, and

${ }^{a}$ Shanghai Key Laboratory of New Drug Design, Shanghai Key Laboratory of Chemical Biology, School of Pharmacy, State Key Laboratory of Bioengineering Reactor, East China University of Science and Technology, 130 Meilong Road, Shanghai 200237, China.E-mail: kylou@ecust.edu.cn

${ }^{b}$ School of Pharmacy, Shaanxi Institute of International Trade \& Commerce, Xi'an 712046, China

'Department of Chemistry and Chemical Biology, University of New Mexico, Albuquerque, NM 87131-0001, USA. E-mail: wwang@unm.edu

$\dagger$ Electronic supplementary information (ESI) available: Details of the synthetic procedures, additional fluorescence spectra, and NMR and MS spectra. See DOI: $10.1039 / \mathrm{c} 8 \mathrm{ra02138 \textrm {d }}$

$\$$ These two authors contributed equally. selective fluorescent probe for Cys based on a dual photoinduced electron transfer (РеT) and photo-induced intramolecular charge transfer (ICT) quenching mechanism. ${ }^{28}$ Different from many other maleimide-based fluorescent probes that only undergo a PeT mechanism, ${ }^{15}$ the additional ICT quenching mechanism keeps the 1,8-naphthalimide (NAP) fluorophore in the thiol-Michael adduct in a low fluorescence emission state due to the strong electron-withdrawing effects of the succinimide group at its 4-position. Then, a subsequent transcyclization step, involving the formation of a sixmembered thiomorpholinone ring and cleavage of a fivemembered succinimide ring, converts the non-fluorescent thiol-Michael adduct into the major fluorescent product, in which the ICT quenching is removed, resulting in a drastic fluorescence turn-on response. ${ }^{28} \mathrm{~A}$ similar transcyclization process and the simultaneous removal of ICT quenching allowed us to design a NAP-based turn-on fluorescent probe for $\gamma$-glutamyltranspeptidase ${ }^{29}$ and a coumarin-based turn-on fluorescent probe with dual recognition groups and dual cyclization for the selective detection of Cys. ${ }^{30}$ In addition, another
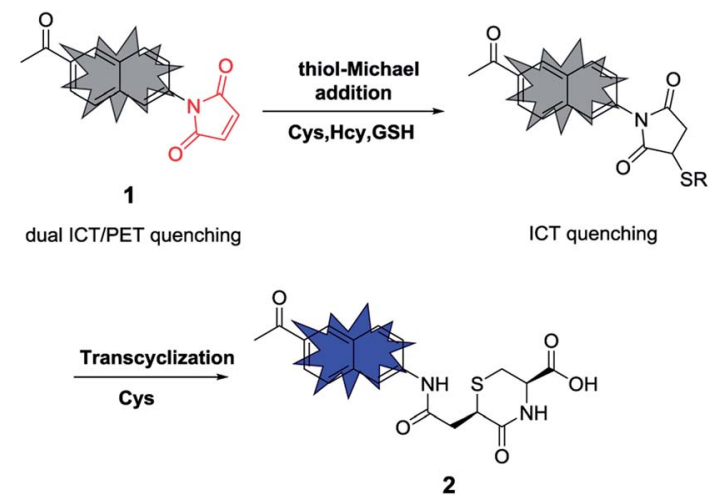

Fig. 1 Design rationale of the fluorescent probe 1 for the selective turn-on detection of Cys over Hcy and GSH. 

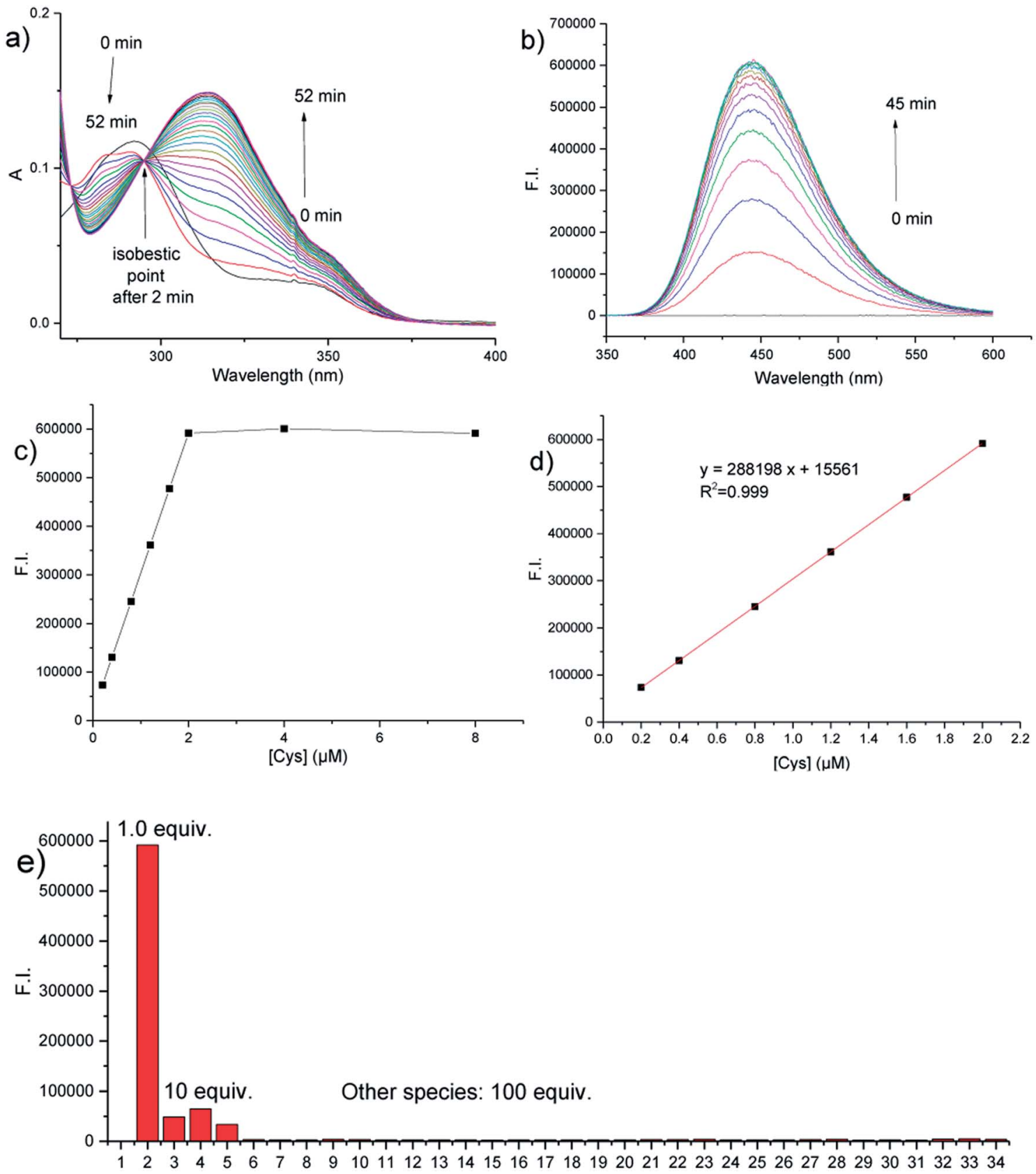

Fig. 2 (a) Time-dependent UV-vis spectra of probe $1(10 \mu \mathrm{M})$ upon the addition of 1 equiv. of Cys (a spectrum was recorded every 2 minutes); (b) time-dependent fluorescence emission spectra of probe $1(2 \mu \mathrm{M})$ upon the addition of 1 equiv. of Cys (a spectrum was recorded every 3 minutes); (c) time-dependent fluorescence emission intensity at $446 \mathrm{~nm}$ of probe $1(2 \mu \mathrm{M})$ upon addition of Cys ( 0 to 4 equiv.); (d) a linear relationship of the fluorescence intensity at $446 \mathrm{~nm}$ versus the Cys concentration $(0.2-2.0 \mu \mathrm{M})$; (e) fluorescence response of probe $1(2 \mu \mathrm{M})$ at $446 \mathrm{~nm}$ toward various species in PBS buffer (10 mM, pH 7.4): (1) blank; (2) Cys; (3) Hcy; (4) GSH; (5) NAC; (6) valine; (7) glycine; (8) isoleucine; (9) lysine; (10) leucine; (11) histidine; (12) asparagine; (13) methionine; (14) proline; (15) serine; (16) alanine; (17) threonine; (18) arginine; (19) glutamine; (20) aspartic acid; (21) glutamic acid; (22) tyrosine; (23) tryptophan; (24) phenylalanine; (25) glucose; (26) $\mathrm{H}_{2} \mathrm{O}_{2} ;(27) \mathrm{Na}^{+} ;(28) \mathrm{K}^{+} ;(29) \mathrm{Ca}^{2+}$; (30) $\mathrm{Mg}^{2+}$; (31) $\mathrm{Fe}^{3+}$; (32) $\mathrm{Fe}^{2+}$; (33); $\mathrm{Cu}^{2+} ;$ (34) $\mathrm{Zn}^{2+}$ (All measurements were made in $10 \mathrm{mM} \mathrm{PBS}$ buffer, $\mathrm{pH} 7.4,25^{\circ} \mathrm{C}$, and $\lambda_{\mathrm{ex}}=314 \mathrm{~nm}$ ).

NAP-based dual PeT/ICT probe was recently reported by Meka and Heagy for the detection of hydrogen sulfide, although two recognition groups instead of one were adopted in their probe to achieve the dual quenching mechanism. ${ }^{31}$

Our previous work and that of other groups has demonstrated that the combination of PeT and ICT mechanisms is particularly suitable for the design of fluorescent probes with a significant fluorescence turn-on response. ${ }^{30-33}$ However, many of these probes have a short excitation wavelength in the UV or visible range, which is not optimal for biological applications due to enhanced phototoxicity and/or autofluorescence..$^{34,35}$ Considering that two-photon fluorescence imaging has advantages such as the excitation process being carried out by a nearinfrared (NIR) laser that has a reduced cell toxicity and low 
fluorescence background, ${ }^{36}$ in this work, we aimed to introduce a similar dual PeT/ICT quenching mechanism to the known two-photon fluorophore 6-acyl-2-naphthylamine ${ }^{37-39}$ in order to design a simple maleimide-based two-photon fluorescent probe, $\mathbf{1}$, for the selective detection of Cys over Hcy and GSH. It was also tested to determine whether it is a turn-on fluorescent probe with high sensitivity and selectivity, which reacts with Cys via a fast two-step thiol-Michael addition and transcyclization cascade reaction. ${ }^{28}$ The structure of probe 1 is shown in Fig. 1. It has a maleimide group at its 2-position, which promotes the PeT quenching effect. It also has an additional electronwithdrawing methylcarbonyl group at its 6-position to ensure a pull-pull ICT quenching effect.

Probe 1 was conveniently synthesized from 6-acyl-2naphthylamine $(3)^{39}$ in a two-step process with a total yield of $38 \%$ (see Scheme $\mathrm{S} 1$ in the ESI $\dagger$ ). First, the amine 3 was reacted with maleic anhydride to form the maleic amide acid 4 . Then, the amide acid $\mathbf{4}$ was cyclized to afford the maleimide $\mathbf{1}$ in the presence of acetic anhydride (see the $\mathrm{ESI} \dagger$ for more details).

We then investigated the absorption and fluorescence emission response of the probe towards just 1 equiv. of Cys. The time-dependent absorption spectra upon the addition of 1 equiv. of Cys are shown in Fig. 2a. Probe $\mathbf{1}$ has a maximum absorption peak at $292 \mathrm{~nm}$. Upon addition of Cys, the maximum absorption peak shifts to $314 \mathrm{~nm}$, a red-shift of $22 \mathrm{~nm}$. Notably, an isosbestic point can be seen at $295 \mathrm{~nm}$ after $2 \mathrm{~min}$, indicating the formation of an intermediate within 2 min, which is then converted into the final product. The UV spectral changes supported the presence of a proposed cascade reaction sequence for the fast formation of a thiol-Michael adduct intermediate, which then underwent a relatively slow intramolecular transcyclization process to give the final product. From time-dependent fluorescence emission studies (Fig. 2b), probe 1 was found to have almost no fluorescence emission due to dual PeT and ICT quenching effects. Upon the addition of 1 equiv. of Cys, a drastic turn-on fluorescence response (a $>3000$ fold increase) was observed at $446 \mathrm{~nm}$ (see Fig. S1b in the ESI†). The fluorescence intensity at $446 \mathrm{~nm}$ reached its maximum value after around $30 \mathrm{~min}$ indicating that the cascade reaction finished in about $30 \mathrm{~min}$ (Fig. 2b, and S2a in the ESI $\dagger$ ). The pseudo-first-order reaction kinetic constant based on the fluorescence enhancement was calculated as $0.123 \mathrm{~min}^{-1}$ (half-time $=5.64 \mathrm{~min}$, Fig. S2b in the ESI $\dagger$ ), indicating an overall fast cascade reaction. Fluorescence titration experiments using an increasing amount of Cys from 0 to 4.0 equiv. over $30 \mathrm{~min}$ showed a steady increase in the fluorescence intensity and the maximum intensity was reached at exactly 1.0 equiv. of Cys. Further Cys addition did not increase the fluorescence intensity, indicating that probe 1 reacts with Cys in a $1: 1$ molar ratio (Fig. $2 \mathrm{c}$ and $\mathrm{S} 3$ in the ESI $\dagger$ ), which was also supported by the Job plot (see Fig. S4 in the ESI $\dagger$ ). From the linear relationship of the fluorescence intensity at $446 \mathrm{~nm}$ versus the Cys concentrations, the detection limit of probe $\mathbf{1}$ (2 $\mu \mathrm{M})$ for Cys was calculated as $1.4 \mathrm{nM}(\mathrm{S} / \mathrm{N}=3$, Fig. 3d), indicating that $\mathbf{1}$ is a highly sensitive probe for Cys. Moreover, the probe showed excellent selectivity for the detection of Cys over many other species (Fig. 2e, and S5 in the ESI $\dagger$ ), including the

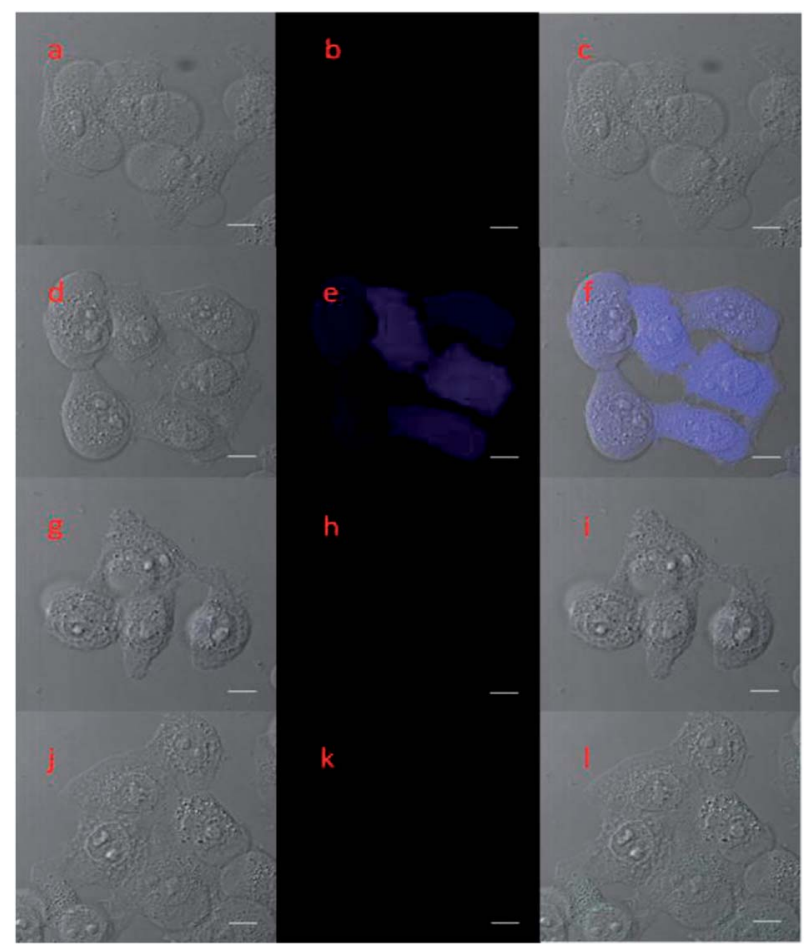

Fig. 3 Two-photon fluorescence images (b, e, h, k) of HeLa cells collected at $410-510 \mathrm{~nm}$ (blue to cyan-blue, $\lambda_{\text {ex }}=690 \mathrm{~nm}$ ), the corresponding bright field view $(a, d, g, j)$, and overlap of the fluorescence channel and the bright field view $(\mathrm{c}, \mathrm{f}, \mathrm{i}, \mathrm{l})$ after different treatments: $(\mathrm{a}-\mathrm{c})$ the cells were pretreated with $0.5 \mathrm{mM}$ of $\mathrm{N}$ ethylmaleimide (NEM) for 30 min and then incubated with $10 \mu \mathrm{M}$ of probe 1 for $30 \mathrm{~min}$; (d-f) cells were first pretreated with $0.5 \mathrm{mM}$ of NEM for $30 \mathrm{~min}$, then after addition of $1 \mathrm{mM}$ of Cys were incubated for $30 \mathrm{~min}$, and finally, incubated with $10 \mu \mathrm{M}$ of probe 1 for $30 \mathrm{~min}$ (scale bar $=10 \mu \mathrm{m})$; the conditions for $(\mathrm{g}-\mathrm{i})$ and $(j-l)$ were similar to those of $(d-f)$, except that $10 \mu \mathrm{M}$ of Hcy and $10 \mu \mathrm{M}$ of GSH were used instead of $10 \mu \mathrm{M}$ of Cys.

structurally similar thiols Hcy, GSH, and $N$-acetylcysteine (NAC). The fluorescence intensity at $446 \mathrm{~nm}$ for 1 equiv. of Cys was significantly higher (12.2-fold, 9.1-fold, and 17.7-fold, respectively) than that of 10 equiv. of Hcy, GSH, or NAC. To further confirm the reaction mechanism, the reaction product, 2, from the reaction of probe $\mathbf{1}$ with Cys, was isolated and its structure was confirmed using ${ }^{1} \mathrm{H}$ nuclear magnetic resonance (NMR) spectroscopy, ${ }^{13} \mathrm{C}$ NMR spectroscopy, 2D-rotating-frame nuclear Overhauser effect spectroscopy (ROESY), and high-resolution mass spectrometry (HRMS) (see the ESI for more details $\dagger$ ). The fluorescence quantum yields of probe 1 and product 2 were measured as 0.002 and 0.782 , respectively (see the ESI for more details $\dagger$ ). Therefore, the formation of the transcyclization product 2 was determined to be responsible for the observed fluorescence turn-on response. For the other thiols, the transcyclization steps of the thiol-Michael adducts were much slower, resulting in the observed high selectivity. Overall, we have shown here that probe $\mathbf{1}$ is a highly sensitive and selective turn-on fluorescent probe for Cys.

Encouraged by the fast, selective, and sensitive in vitro fluorescence response of probe $\mathbf{1}$ for the detection of Cys, we further evaluated its potential use as a two-photon imaging 
agent for Cys in biological systems, such as in living cells. The fluorescence response of probe $\mathbf{1}$ towards Cys at different $\mathrm{pH}$ values was evaluated and a suitable $\mathrm{pH}$ range for Cys detection was determined to be 7.0 to 10.0 , which is a good range for cell imaging applications because physiological conditions have a pH of around 7.4 (see Fig. S7 in the ESI $\dagger$ ). HeLa cells were then pretreated with $\mathrm{N}$-ethylmaleimide (NEM, $0.5 \mathrm{mM}$ ) for $30 \mathrm{~min}$ to remove the endogenous cellular thiols, and incubated with Cys (1 $\mathrm{mM})$, Hcy ( $1 \mathrm{mM})$, or GSH $(1 \mathrm{mM})$, respectively for $30 \mathrm{~min}$ to increase the specific thiol levels. The samples were then further incubated with probe $1(10 \mu \mathrm{M})$ for $30 \mathrm{~min}$ and were then washed with PBS buffer before two-photon fluorescence cell images and the corresponding bright-field view images were taken (Fig. 3(d-l)). Control images were also taken for samples pretreated with NEM $(0.5 \mathrm{mM})$ and then incubated with probe 1 $(10 \mu \mathrm{M})$ (Fig. 3a-c). Only cells pretreated with NEM and then Cys showed a distinctive blue fluorescence (Fig. 3e). The above cell imaging studies clearly demonstrated that probe $\mathbf{1}$ is capable of the selective detection and imaging of intracellular Cys over Hcy and GSH in living cells by two-photon fluorescence imaging with low background fluorescence interference.

\section{Conclusions}

We have demonstrated that $N$-(6-acyl-2-naphthayl)-maleimide (1) is a highly sensitive and selective simple fluorescent probe for the turn-on detection of Cys over other biothiols, including Hcy and GSH, with a detection limit of as low as $1.4 \mathrm{nM}$. The probe has very low fluorescence background due to a dual quenching mechanism of PeT and ICT. Upon reaction with Cys via a thiol-Michael addition and intramolecular transcyclization cascade reaction, the maleimide group at the 2-position was replaced by a better electron-donating succinamide group, resulting in more than a 3000-fold fluorescence enhancement due to the removal of dual quenching effects. The probe provided additional evidence of the rational design of a Cys-selective fluorescent probe by tuning the fluorescence turn-on response only at the Cys-selective transcyclization step in a two-step thiol-Michael-addition-transcyclization cascade reaction via a dual PeT/ICT mechanism, suggesting that the design of a $N$-fluorophore-substituted maleimide with dual PeT/ICT quenching, initially demonstrated on the 1,8-naphthalimide fluorophore platform ${ }^{28}$ is a rather general approach for obtaining a Cys-selective turn-on fluorescent probe with high selectivity and sensitivity. Moreover, the probe was successfully applied in the two-photon imaging of intracellular Cys in cultured HeLa cells excited by a near-infrared $690 \mathrm{~nm}$ laser.

\section{Conflicts of interest}

There are no conflicts to declare.

\section{Acknowledgements}

This work was supported by the National Natural Science Foundation of China (Grant No. 21577037, K. L.) and the East China University of Science and Technology (start-up funds, W. W.).

\section{Notes and references}

1 R. O. Ball, G. Courtney-Martin and P. B. Pencharz, J. Nutr., 2006, 136, 1682S.

2 K. G. Reddie and K. S. Carroll, Curr. Opin. Chem. Biol., 2008, 12, 746.

3 X. F. Wang and M. S. Cynader, J. Neurosci., 2001, 21, 3322.

4 D. H. Baker and G. L. Czarnecki-Maulden, J. Nutr., 1987, 117, 1003.

5 J. Selhub, Annu. Rev. Nutr., 1999, 19, 217.

6 H. Sies, Free Radical Biol. Med., 1999, 27, 916.

7 S. Shahrokhian, Anal. Chem., 2001, 73, 5972.

8 S. Seshadri, A. Beiser, J. Selhub, P. F. Jacques, I. H. Rosenberg, R. B. D'Agostino, P. W. F. Wilson and P. A. Wolf, N. Engl. J. Med., 2002, 346, 476.

9 A. A. Mangoni and S. H. D. Jackson, Am. J. Med., 2002, 112, 556.

10 D. M. Townsend, K. D. Tew and H. Tapiero, Biomed. Pharmacother., 2003, 57, 145.

11 W. H. Wang, O. Rusin, X. Y. Xu, K. K. Kim, J. O. Escobedo, S. O. Fakayode, K. A. Fletcher, M. Lowry, C. M. Schowalter, C. M. Lawrence, F. R. Fronczek, I. M. Warner and R. M. Strongin, J. Am. Chem. Soc., 2005, 127, 15949.

12 T. Inoue and J. R. Kirchhoff, Anal. Chem., 2002, 74, 1349.

13 W. Wang, L. Li, S. Liu, C. Ma and S. Zhang, J. Am. Chem. Soc., 2008, 130, 10846.

14 F.-J. Huo, Y.-Q. Sun, J. Su, J.-B. Chao, H.-J. Zhi and C.-X. Yin, Org. Lett., 2009, 11, 4918.

15 X. Chen, Y. Zhou, X. Peng and J. Yoon, Chem. Soc. Rev., 2010, 39, 2120.

16 H. S. Jung, X. Chen, J. S. Kim and J. Yoon, Chem. Soc. Rev., 2013, 42, 6019.

17 H. Chen, Y. Tang and W. Lin, TrAC, Trends Anal. Chem., 2016, 76, 166.

18 Y. Yang, Q. Zhao, W. Feng and F. Li, Chem. Rev., 2013, 113, 192.

19 X. Qian, Y. Xiao, Y. Xu, X. Guo, J. Qian and W. Zhu, Chem. Commun., 2010, 46, 6418.

20 Y. Tang, D. Lee, J. Wang, G. Li, J. Yu, W. Lin and J. Yoon, Chem. Soc. Rev., 2015, 44, 5003.

21 L.-Y. Niu, Y.-Z. Chen, H.-R. Zheng, L.-Z. Wu, C.-H. Tung and Q.-Z. Yang, Chem. Soc. Rev., 2015, 44, 6143.

22 O. Rusin, N. N. St Luce, R. A. Agbaria, J. O. Escobedo, S. Jiang, I. M. Warner, F. B. Dawan, K. Lian and R. M. Strongin, J. Am. Chem. Soc., 2004, 126, 438.

23 X. Yang, Y. Guo and R. M. Strongin, Angew. Chem., Int. Ed., 2011, 50, 10690.

24 L. Long, W. Lin, B. Chen, W. Gao and L. Yuan, Chem. Commun., 2011, 47, 893.

25 L.-Y. Niu, Y.-S. Guan, Y.-Z. Chen, L.-Z. Wu, C.-H. Tung and Q.-Z. Yang, J. Am. Chem. Soc., 2012, 134, 18928. 
26 L.-Y. Niu, Y.-S. Guan, Y.-Z. Chen, L.-Z. Wu, C.-H. Tung and Q.-Z. Yang, Chem. Commun., 2013, 49, 1294.

27 J. Liu, Y.-Q. Sun, H. Zhang, Y. Huo, Y. Shi and W. Guo, Chem. Sci., 2014, 5, 3183.

28 X. Li, Y. Zheng, H. Tong, R. Qian, L. Zhou, G. Liu, Y. Tang, H. Li, K. Lou and W. Wang, Chem.-Eur. J., 2016, 22, 9247.

29 H. Tong, Y. Zheng, L. Zhou, X. Li, R. Qian, R. Wang, J. Zhao, K. Lou and W. Wang, Anal. Chem., 2016, 88, 10816.

30 H. Tong, J. Zhao, X. Li, Y. Zhang, S. Ma, K. Lou and W. Wang, Chem. Commun., 2017, 53, 3583.

31 R. K. Meka and M. D. Heagy, J. Org. Chem., 2017, 82, 12153.

32 L. He, B. Dong, Y. Liu and W. Lin, Chem. Soc. Rev., 2016, 45, 6449.
33 H. Zhang, C. Zhang, R. Liu, L. Yi and H. Sun, Chem. Commun., 2015, 51, 2029.

34 Y. Matsumura and H. N. Ananthaswamy, Toxicol. Appl. Pharmacol., 2004, 195, 298.

35 M. Monici, Biotechnol. Annu. Rev., 2005, 11, 227.

36 H. M. Kim and B. R. Cho, Chem. Rev., 2015, 115, 5014.

37 Z. Xiang and L. Wang, J. Org. Chem., 2011, 76, 6367.

38 S. Singha, D. Kim, B. Roy, S. Sambasivan, H. Moon, A. S. Rao, J. Y. Kim, T. Joo, J. W. Park, Y. M. Rhee, T. Wang, K. H. Kim, Y. H. Shin, J. Jung and K. H. Ahn, Chem. Sci., 2015, 6, 4335. 39 M. Wen, X. Guo, P. Sun, L. Xiao, J. Li, Y. Xiong, J. Bao, T. Xue, L. Zhang and C. Tian, Chem. Commun., 2015, 51, 8153. 